\title{
Kinematic characteristics of grip force in patients with cervical spondylosis
}

\author{
Bumsuk Lee ${ }^{a}$, Naoto Noguchi ${ }^{b}$, Daiki Kakiage ${ }^{c}$, Tsuneo Yamazaki $^{a}$ \\ ${ }^{a}$ Graduate School of Health Sciences, Gunma University, Gunma, Japan \\ ${ }^{b}$ Department of Rehabilitation, Harunaso Hospital, Gunma, Japan \\ 'Division of Rehabilitation Medicine, Gunma University Hospital, Gunma, Japan
}

Objective: The aim of this study was to objectively evaluate sensory disturbance in cervical spondylosis using grip force and investigate the relationship between the grip force and upper extremity function.

Design: Cross-sectional study.

Methods: Eleven cervical spondylosis patients with paresthesia conducted grip and lift tasks using a precision grip with the tips of the thumb and index finger on either side. The sum of the grip force used during the first four seconds was calculated and defined as the total grip force. The cutaneous pressure threshold of the fingers, the pinch power, the grip power and three subtests of the Simple Test for Evaluating Hand Function (STEF) were also assessed. Correlations between the total grip force and cutaneous pressure threshold, pinch power, grip power, and STEF subtest times were evaluated.

Results: We found that the total grip force correlated with the cutaneous pressure threshold $(p<0.05)$. Moreover, the total grip force of the dominant thumb correlated with the results of the three STEF subtests $(p<0.05)$. There were no significant correlations between the total grip force and pinch/grip powers.

Conclusions: We found that the total grip force correlated with cutaneous pressure threshold and upper extremity function. The results suggest that the total grip force could serve as an objective index for evaluating paresthesia in cervical spondylosis patients, and that the impaired ability of the upper extremity function is related to grip force coordination.

Key Words: Cervical spondylosis, Grip force, Paresthesia

\section{Introduction}

Gripping and lifting objects is the basis of upper extremity function. Grip force trace indicates successive phases while gripping and lifting objects. According to Johansson and Flanagan [1], grip force trace can be broken down into distinct phases: the reach phase when the digits contact the object; the load phase when contact is broken between the object and the surface of the table; the lift phase which is the time interval between the object lifting off the surface and the object approaching the goal height; the hold phase which involves holding the object in the air; the replace phase and the unload phase. If objects are heavy and/or slippery, great- er grip force is needed to avoid letting the objects slip.

Blennerhassett et al. [2] compared grip force application in stroke patients and matched control subjects, and found fluctuating irregular forces and reduced adaptation of the safety margin in stroke patients. The raised grip force was considered to be a compensatory strategy to maintain grip when sensorimotor processes are deficient. Seo et al. [3] found that the magnitude of the paretic fingers' grip force at the digits was significantly deviated in comparison with asymptomatic hands. Müller and Dichgans [4] reported that cerebellar patients showed an elevated pinch force level at the start of lift force. It is also known that grip force is influenced by sensory and sensorimotor systems. Johansson and

Received: 19 October, 2015 Revised: 4 December, 2015 Accepted: 7 December, 2015

Corresponding author: Bumsuk Lee

Graduate School of Health Sciences, Gunma University, 3-39-22, Showa-machi, Maebashi, Gunma 371-8514, Japan

Tel: 81-27-220-8954 Fax: 81-27-220-8999 E-mail: leebumsuk@gunma-u.ac.jp

(c) This is an Open-Access article distributed under the terms of the Creative Commons Attribution Non-Commercial License (http://creativecommons.org/licens es/by-nc/4.0) which permits unrestricted non-commercial use, distribution, and reproduction in any medium, provided the original work is properly cited.

Copyright @ 2015 Korean Academy of Physical Therapy Rehabilitation Science 
Westling [5] demonstrated that grip force was increased by local cutaneous anesthesia, and considered that this force change was related to cutaneous afferent input.

Cervical spondylosis is caused by unspecified degenerative changes, and clinical characteristics such as pain, stiffness, numbness and radicular pain to the arms and fingers are presented [6]. Moreover, paresthesia of the upper extremity is also a common radicular symptom observed. Sensory symptoms are assessed through many evaluations such as the Nurick-score, Japanese orthopaedic association score and Cooper myelopathy scale [7]. Moreover, Persson et al. [8] used the level of upper extremity paresthesia as an outcome of surgical treatment. However, in many cases the assessment of sensory symptoms is based on the patients' perceived severity or an approximated sensory test. Paresthesia of the upper extremity in cervical spondylosis has not yet been adequately evaluated.

The aim of this study was to objectively evaluate sensory disturbance in cervical spondylosis using grip force. The kinematic characteristics of the grip force trace while gripping and lifting objects were monitored. Moreover, we also investigated the relationship between the grip force and upper extremity function.

\section{Methods}

Eleven male inpatients (mean age, 67.2 \pm 6.5 years) participated in the study. All patients were diagnosed as having cervical spondylotic myelopathy ( 6 patients) or ossification of posterior longitudinal ligament (5 patients), and were waiting for surgical operations. Handedness was evaluated with the Edinburgh Handedness Inventory, and all patients were right-handed. Informed consents were obtained prior to the study. The study was approved by the Research Ethics Committee of Harunaso Hospital.

An iron cube $(250 \mathrm{~g}, 31 \times 31 \times 31 \mathrm{~mm})$ was custom manufactured for this study. Two pressure sensors (FlexiForce ${ }^{\mathrm{TM}}$; Tekscan Inc., South Boston, MA, USA; sensing area diameter, $9.5 \mathrm{~mm}$; measurement range, $0-110 \mathrm{~N}$ ) were attached to the surfaces of the cube to measure the grip force at each contact surface. The sensor acts as a resistor in the electrical circuit. When the sensor is unloaded, the resistance is very high. When force is applied to the sensor, this resistance decreases. The resistance can be read by connecting an ohmmeter to the outer two pins of the sensor connector and applying force to the sensing area. The resistance converted voltage was converted from analog-to-digital by using a data logger system (DL 3100; S\&ME Inc., Tokyo, Japan). Data were sampled at $1,000 \mathrm{~Hz}$ with a resolution of 16-bit. The reliability and validity were reported in previous experiments $[9,10]$.

The patients sat in a chair in front of a desk that supported the cube. Verbal instructions were given to grip the cube using the thumb and index finger, lift approximately $10 \mathrm{~cm}$, and hold for 5-7 seconds. Before lifting, patients were allowed to touch the cube slightly without exceeding $0.5 \mathrm{~N}$. Ten lifts were performed in each hand and the interval between lifts was approximately five seconds. Half of the patients performed the first session with the dominant hand, and vice versa.

Some studies with healthy subjects have reported that there is maximum grip force in the lift phase [1,11]. Our previous study also used maximum grip force to investigate dual task interference in a grip and lift task [12]. However, it was not possible to identify maximum grip force in many patients in this study. Therefore, we calculated the sum of the grip force used during the first four seconds, and defined it as the total grip force.

The cutaneous pressure threshold of the central areas of the thumb and index finger pulps was assessed with Touch Test Sensory Evaluators (North Coast Medical Inc., Morgan Hill, CA, USA), which apply a standard calibrated force of $0.07,0.4,2.0,40$ and $300 \mathrm{~g}$. The lowest perceived monofilament is considered normal and higher monofilament forces indicate the severity of the sensory disturbance.

The pinch power between thumb and index finger was measured with the JAMAR Hydraulic Pinch Gauge (Petterson Medical, Warrenville, IL, USA), and the grip power was measured with the Smedley Grip Tester (Gopher, Owatonna, MN, USA). The upper extremity function was evaluated using three subtests (number 8, 9, and 10) of the Simple Test for Evaluating Hand Function (STEF). These three subtests require patients to pinch objects with the thumb and index finger. For example, in subtest 10, patients reach and pinch a pin $(0.3 \mathrm{~cm}$ in diameter and $4 \mathrm{~cm}$ long $)$ and then bring the pin into a target space as quickly as possible, repeating this process six times (Figure 1). Time to complete each subtest was used for statistical analysis.

The total grip force was compared among the four fingers using one way ANOVA. Pearson's correlation was calculated between the total grip force and cutaneous pressure threshold (11 patients $\times 4$ fingers). Moreover, Pearson's correlations between the total grip force in each finger and rest of the measurements (pinch power, grip power and STEF 

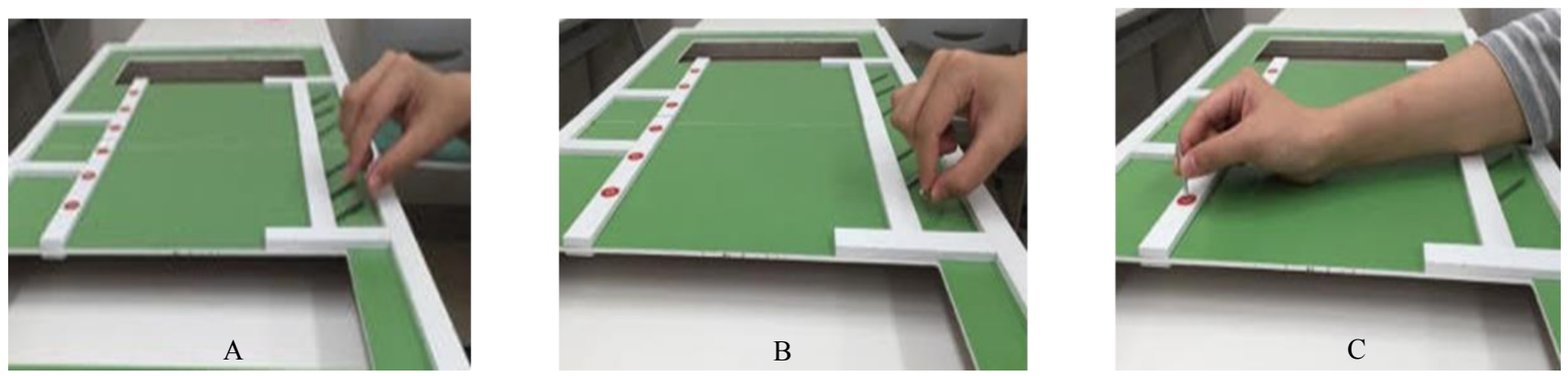

Figure 1. Subtest 10 of the Simple Test for Evaluating Hand Function. Patients reach (A) and pinch a pin (B) and bring the pin into a target space $(\mathrm{C})$ as quickly as possible, and repeat this process six times.
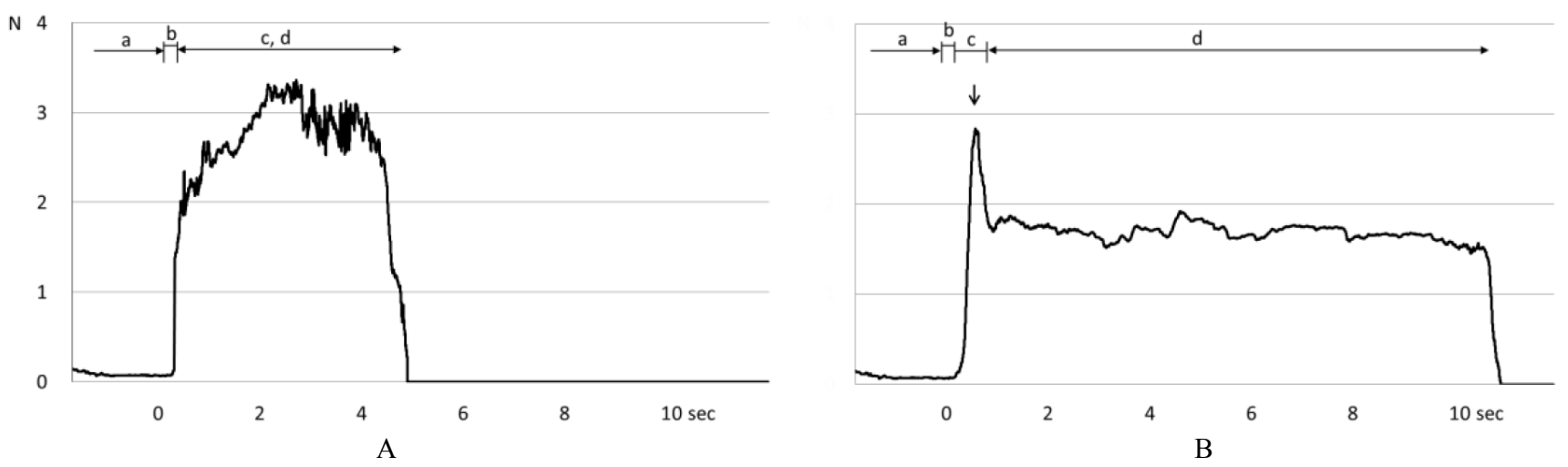

Figure 2. Grip force trace while performing grip and lift task. (A) A representative patient in the study. (B) A healthy subject in our previous study. Revised from the article of Lee et al. (Kitakanto Med J 2014;64:309-12) [12]. Reach (a), load (b), lift (c), and hold (d) phases, and maximum grip force $(\downarrow)$ were identified in Figure 2B. However, in Figure 2A the maximum grip force was not identified, and grip force trace was not stable relatively.

subtest times) were also calculated. The statistical software SPSS ver. 21.0J for Windows (SPSS Japan Inc., Tokyo, Japan) was used for the analyses, and $p<0.05$ were considered significant.

\section{Results}

Figure 2 shows the grip force trace for a representative patient in the study (Figure 2A) and that of a healthy subject in our previous study (Figure 2B) [12]. In Figure 2B, Reach (a), load (b), lift (c) and hold (d) phases, and maximum grip force $(\downarrow)$ were identified. However, as shown in Figure 2A, maximum grip force was not identified in cervical spondylosis patients, and grip force trace was relatively unstable.

The total grip forces were 6,042 $\pm 2,905$ (N/4 seconds) for dominant thumb, 5,595 $\pm 4,214$ for dominant index finger, $5,739 \pm 4,839$ for non-dominant thumb and 7,075 $\pm 6,895$ for non-dominant index finger. Averaged across all 11 patients, the pinch power was $6.0 \pm 1.2 \mathrm{~kg} / 6.4 \pm 1.6 \mathrm{~kg}$, the grip power
Table 1. Pearson's correlation between the total grip force (N/4 seconds) and cutaneous pressure threshold $\quad(\mathrm{N}=11)$

\begin{tabular}{cc}
\hline & Total grip force \\
\hline Cutaneous pressure threshold & $0.41^{* *}$ \\
${ }^{* *} p<0.01$. &
\end{tabular}

was $33.1 \pm 6.0 \mathrm{~kg} / 33.3 \pm 6.7 \mathrm{~kg}$ (dominant/non-dominant). Times to complete each subtest (dominant/non-dominant) were $12.1 \pm 3.5$ seconds/12.7 \pm 4.1 seconds for subtest 8 , $12.7 \pm 2.7$ seconds/ $13.1 \pm 3.4$ seconds for subtest 9 and $14.9 \pm$ 4.0 seconds $/ 15.3 \pm 4.2$ seconds for subtest 10 .

When the total grip force was compared among the four fingers using one way ANOVA, there was no significant difference. As shown in Table 1, there were positive correlations between the total grip force and cutaneous pressure threshold $(\mathrm{r}=0.41, p<0.01)$. Although we did not observe significant correlation in pinch power or grip power, there was a positive correlation between the total grip force of the 
Table 2. Pearson's correlations between the total grip force (N/4 seconds) in each finger and the rest of the measurements ( $N=11)$

\begin{tabular}{|c|c|c|c|c|c|}
\hline & & \multicolumn{4}{|c|}{ The total grip force } \\
\hline & & \multicolumn{2}{|c|}{ Dominant } & \multicolumn{2}{|c|}{ Non-dominant } \\
\hline & & Thumb & Index finger & Thumb & Index finger \\
\hline \multirow[t]{2}{*}{ Pinch power $(\mathrm{kg})$} & Dominant & -0.46 & -0.37 & - & - \\
\hline & Non-dominant & - & - & -0.22 & -0.27 \\
\hline \multirow[t]{2}{*}{ Grip power (kg) } & Dominant & -0.40 & -0.03 & - & - \\
\hline & Non-dominant & - & - & 0.03 & 0.19 \\
\hline \multirow[t]{2}{*}{ STEF subtest 8 (s) } & Dominant & $0.76^{* *}$ & 0.52 & - & - \\
\hline & Non-dominant & - & - & 0.10 & 0.20 \\
\hline \multirow[t]{2}{*}{ STEF subtest 9 (s) } & Dominant & $0.65^{*}$ & 0.21 & - & - \\
\hline & Non-dominant & - & - & -0.01 & 0.03 \\
\hline \multirow[t]{2}{*}{ STEF subtest 10 (s) } & Dominant & $0.64^{*}$ & 0.22 & - & - \\
\hline & Non-dominant & - & - & -0.11 & -0.05 \\
\hline
\end{tabular}

STEF: Simple Test for Evaluating Hand Function. ${ }^{*} p<0.05 ;{ }^{* *} p<0.01$.

dominant thumb and the STEF subtest times $(\mathrm{r} \geq 0.64, p \leq$ 0.05) (Table 2).

\section{Discussion}

We found that the total grip force correlated with cutaneous pressure threshold and upper extremity function. Although a previous study already reported increased grip force in the digital nerves block of healthy subjects [5], to our knowledge, this study provides the first assessment of grip force in cervical spondylosis patients with paresthesia. We also found that there was no correlation between the total grip force and pinch/grip power.

Before the experiment, based on our previous study [12], we had expected that maximum grip force would also be a proper component in this study. However, as shown in Figure 2A, maximum grip force was not identified in many patients. In other words, the patients increased and decreased the grip force multiple times in order to avoid letting the cube slip during the holding phase. This may be explained by upper extremity paresthesia in cervical spondylosis. In healthy subjects, sensory information related to weight and friction is sent to the central nervous system through afferent nerve fibers and used in grip force coordination. Compared to healthy subjects, the lack of sensory information in cervical spondylosis patients caused incomplete grip force coordination. Consequently, increasing/ decreasing grip force occurred multiple times during the holding phase. The influence of paresthesia in the results was in good accordance with the previous study [5].
The influence of paresthesia was also found in the total grip force. The fingers, which are in severe paresthesia, tend to increase grip force. Increased grip force has been reported in patients with carpel tunnel syndrome [13], chronic somatosensory deafferentation [14] and congenital insensitivity to pain [15]. These previous studies revealed that the lack of sensory feedback caused a deficit of grip force coordination, sometimes even if the motor system is intact. It is not difficult to imagine that the patients with severe paresthesia increase grip force to compensate for incomplete grip force coordination. Therefore, we suggest that the total grip force is a proper component for measuring the characteristics of grip force trace in cervical spondylosis patients.

There was a positive correlation between the total grip force of the dominant thumb and the STEF subtest times. We think this correlation implies the specific role of the thumb in the upper extremity function. It is well known that the movement of the thumb underlies all the skilled procedures of which the hand is capable [16]. Especially, the thumb plays a crucial role in the upper extremity movements accompanying a precision pinch. Therefore, the impaired ability of upper extremity function in cervical spondylosis patients should be discussed in relation to decreased grip force coordination of the thumb. It is interesting that only the dominant thumb correlated with upper extremity function. The results are discussed from the aspect of upper limb symmetry. Generally, a dominant arm advantage is known to be the basis of upper limb asymmetries. For example, the dominant arm has advantages in the generation of motor output, including increases in strength, speed, and consistency of movement. 
Dominant hand superiority in the rate of rapid tapping was observed at finger, wrist, and shoulder joints [17]. Asymmetry in our study implies that the capability of grip force coordination is closely related to upper extremity function in the dominant rather than the non-dominant hand.

In contrast with the correlation between the total grip force and the upper extremity function, there was no significant correlation between the total grip force and pinch/ grip power. This may be explained by our experimental design. The patients were asked to grip, lift and hold the cube with minimal force as far as possible. Therefore, the total grip force evaluated how the patients could perform the task without strong muscle contractions. In contrast, pinch/grip power represent strong muscle contractions. This result indicates that the patients understood the aim of the experiment.

On the basis of the above considerations, it seems reasonable to assume that one of the reasons causing increased total grip force in cervical spondylosis patients is paresthesia. Moreover, the impaired ability of upper extremity function in cervical spondylosis patients is related to grip force coordination. However, some limitations of the present study should be acknowledged. Firstly, we did not investigate magnetic resonance imaging (MRI) results. Therefore, it is still unknown as to whether or not the correlation between increased grip force and upper extremity function is related to the degree of cervical spondylosis. A future study with MRI should be considered to clarify the relationship between grip force coordination, upper extremity function and radiological assessment. Secondly, this study was performed with a small number of subjects so the results should be interpreted with caution.

\section{Conflict of Interest}

The authors declared no potential conflicts of interest with respect to the authorship and/or publication of this article.

\section{References}

1. Johansson RS, Flanagan JR. Coding and use of tactile signals from the fingertips in object manipulation tasks. Nat Rev Neurosci 2009;10:345-59.
2. Blennerhassett JM, Carey LM, Matyas TA. Grip force regulation during pinch grip lifts under somatosensory guidance: comparison between people with stroke and healthy controls. Arch Phys Med Rehabil 2006;87:418-29.

3. Seo NJ, Rymer WZ, Kamper DG. Altered digit force direction during pinch grip following stroke. Exp Brain Res 2010;202: 891-901.

4. Müller F, Dichgans J. Dyscoordination of pinch and lift forces during grasp in patients with cerebellar lesions. Exp Brain Res 1994;101:485-92.

5. Johansson RS, Westling G. Roles of glabrous skin receptors and sensorimotor memory in automatic control of precision grip when lifting rougher or more slippery objects. Exp Brain Res 1984;56:550-64.

6. Liang ZH, Di Z, Jiang S, Xu SJ, Zhu XP, Fu WB, et al. The optimized acupuncture treatment for neck pain caused by cervical spondylosis: a study protocol of a multicentre randomized controlled trial. Trials 2012;13:107.

7. Vitzthum HE, Dalitz K. Analysis of five specific scores for cervical spondylogenic myelopathy. Eur Spine J 2007;16:2096-103.

8. Persson LC, Moritz U, Brandt L, Carlsson CA. Cervical radiculopathy: pain, muscle weakness and sensory loss in patients with cervical radiculopathy treated with surgery, physiotherapy or cervical collar. A prospective, controlled study. Eur Spine J 1997; 6:256-66

9. Vecchi F, Freschi C, Micera A, Sabatini AM, Dario P, Sacchetti R. Experimental evaluation of two commercial force sensors for applications in biomechanics and motor control. Paper presented at: 5th Annual Conference of the International Functional Electrical Stimulation Society; 2000 Jul; Aalborg, Danemark.

10. ALmassri AM, Abuitbel MB, WanHasan WZ, Ahmad SA, Sabry AH. Real-time control for robotic hand application based on pressure sensor measurement. Paper presented at: IEEE International Symposium on Robotics and Manufacturing Automation; 2014 Dec 15-16; Kuala Lumpur, Malaysia. p. 80-5.

11. Guillery E, Mouraux A, Thonnard JL. Cognitive-motor interference while grasping, lifting and holding objects. PLoS One 2013; 8:e80125.

12. Lee B, Miyanjo R, Tozato F, Shiihara Y. Dual-task interference in a grip and lift task. Kitakanto Med J 2014;64:309-12.

13. Lowe BD, Freivalds A. Effect of carpal tunnel syndrome on grip force coordination on hand tools. Ergonomics 1999;42:550-64.

14. Hermsdörfer J, Elias Z, Cole JD, Quaney BM, Nowak DA. Preserved and impaired aspects of feed-forward grip force control after chronic somatosensory deafferentation. Neurorehabil Neural Repair 2008;22:374-84.

15. Kawashima N, Abe MO, Iwaya T, Haga N. Abnormal capacity for grip force control in patients with congenital insensitivity to pain. Exp Brain Res 2012;218:579-88.

16. Napier J. Hands. Princeton (NJ): Princetion University Press; 1993. p. 55

17. Todor JI, Kyprie PM, Price HL. Lateral asymmetries in arm, wrist and finger movements. Cortex 1982;18:515-23. 